\title{
ANÁLISE COMPARATIVA DE DISTÂNCIAS NOS SEGUINTES MODELOS: ESFÉRICO E ELIPSOIDAL
}

\author{
Julierme Wagner da Penha \\ Antônio Santana Ferraz \\ Universidade Federal de Viçosa - UFV \\ juliermewagner@yahoo.com.br; ferraz@ufv.br
}

\section{RESUMO}

O conhecimento da distância entre dois pontos situados na superfície terrestre interessa a vários profissionais, principalmente aos engenheiros agrimensores e engenheiros cartógrafos. A Terra não é perfeitamente esférica, sendo a sua forma real segundo Carl Friedrich Gauss (17771855), uma figura conhecida como "geóide", obtida pelo prolongamento do nível médio do mar, homogêneo e supostamente em repouso, através dos continentes. Sendo o geóide uma figura de difícil tratamento matemático, outros sólidos geométricos são utilizados para representação da Terra, notadamente o elipsóide de revolução e a esfera. Neste trabalho procurou-se obter uma rotina para o cálculo de distâncias entre dois pontos da superfície terrestre a partir de suas coordenadas geográficas utilizando-se como modelo matemático da Terra o elipsóide de revolução e, ao mesmo tempo, comparar os valores obtidos para as distâncias entre os mesmos pontos calculadas sobre o modelo esférico. Para esta análise foram selecionadas algumas cidades distribuídas sobre o globo terrestre de maneira a formar rotas em várias direções e magnitudes possibilitando a análise de resultados em várias situações, para se ter uma maior e melhor abrangência experiemental. Os cálculos de distâncias entre dois pontos na esfera são mais simples e foram conduzidos utilizando-se das fórmulas clássicas da trigonometria esférica. Já os cálculos de distâncias na superfície de elipsóide são complexos, envolvendo derivadas e integrais necessitando, portanto, de um software matemático. Os resultados obtidos para o cálculo da distância utilizando-se dos dois modelos são apresentados em valores absolutos e relativos.

\begin{abstract}
Knowledge of the distance between two points is of interest to several professionals, especially surveying and cartographic engineers. The Earth is not perfectly spherical, but rather its surface can be described by the figure known as a geoid, first proposed by Carl Friedrich Gauss (1777-1855). The geoid surface is equivalent to the mean ocean level the Earth, assuming that the oceans are in equilibrium, at rest and extend through the continents. Since the geoid is a difficult figure to treat mathematically, other solid geometric forms are used to represent the Earth's surface, notably the ellipsoid of revolution and the sphere. In this study a routine for calculating the distance between two points on the Earth's surface based on their geographical coordinates was developed using the ellipsoid of revolution as mathematical model of the Earth's surface and the distances calculated were compared to those obtained using the spherical model. The experimental range of the study was purposely widened and enlarged by selecting cities widely distributed around the globe, which allowed for calculating distances in various directions and of various magnitudes. Calculations of distances between two points on the sphere were performed using classical spherical trigonometry formulae. Calculations of distances on the surface of the ellipsoid are more complex than those using the spherical model and required use of mathematical software. The results of distance calculations using the two models are presented as absolute and relative values.
\end{abstract}




\section{INTRODUÇÃO}

A rotação da Terra causa um achatamento nos pólos, assim sendo a sua forma é freqüentemente modelada como um esferóide achatado nos pólos (elipsóide com o raio equatorial maior que o raio polar), ao invés de uma esfera.

O objetivo deste trabalho foi criar uma rotina para calcular distâncias entre 2 pontos da superfície terrestre, com coordenadas geográficas projetadas sobre o elipsóide de revolução, utilizando vários conceitos de plano e comprimento de arco no espaço tridimensional, e comparar a distância entre esses mesmos pontos projetados no modelo esférico, sendo este, muito utilizado na astronomia de campo, através da trigonometria esférica.

Hoje, informações relativas à localização e trajetória são utilizadas em várias áreas como: a geodésia, a navegação oceânica, a navegação aérea, a dinâmica orbital, a transmissão de rádio de grande alcance, o cálculo de trajetórias de mísseis intercontinentais, o cálculo do aquecimento solar em arquitetura, etc.

Alguns conceitos sobre a trigonometria esférica são apresentados, segundo Ferraz (1980):

a) Superfície esférica: é o lugar geométrico dos pontos do espaço que eqüidistam de um ponto interior denominado centro;

b) Círculo máximo: três pontos de uma superfície esférica determinam um plano que a intercepta, segundo um círculo; se tal círculo contiver o centro da esfera, será denominado círculo máximo dessa esfera; em caso contrário será um círculo mínimo (ou círculo menor); as extremidades do diâmetro da esfera, perpendicular ao plano interceptor, são os pólos do círculo (máximo ou mínimo);

c) Triângulo esférico: é a porção da superfície esférica limitada por três arcos de circunferências máximas, menores que $180^{\circ}$ (triângulos eulerianos); ou seja, o triângulo esférico é a figura que se obtém, ligada dois a dois, por meio de arcos de circunferências máximas, com três pontos de uma superfície esférica, não situados sobre a mesma circunferência máxima. $\mathrm{O}$ triângulo esférico então fica constituindo de três ângulos esféricos (A, B e C) e três lados que são arcos de circunferência máxima ( $a, b$ e c $)$, de acordo com a Figura 1.

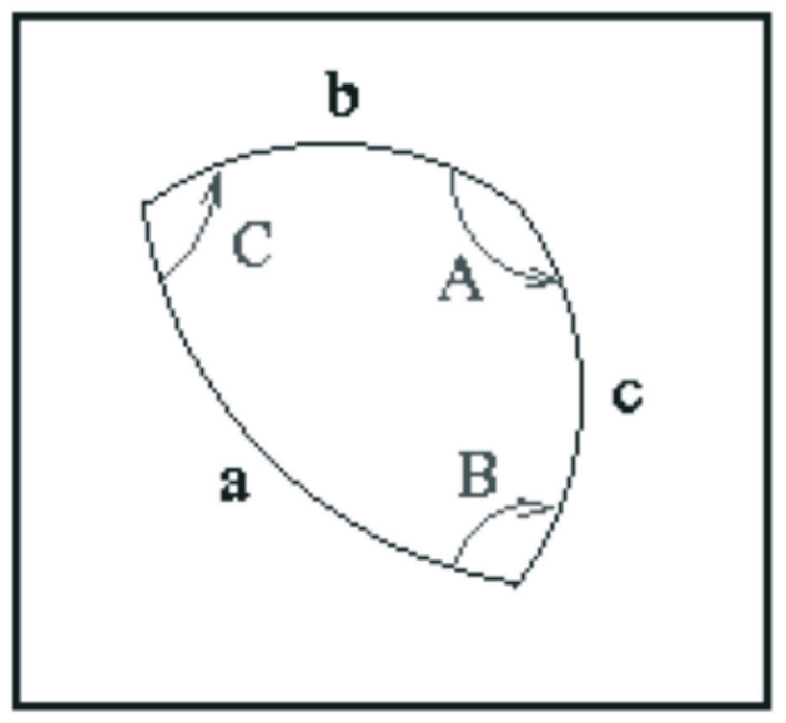

Figura 1. Triângulo esférico.

Sabe-se que no plano a menor distância entre 2 pontos, é uma reta. Porém, se estes postos estiverem na superfície esférica, a menor distância será um arco de circunferência máxima.

Duas de suas propriedades são as seguintes (triangulo esférico): a soma dos ângulos internos de um triângulo esférico é maior que $180^{\circ}$ e menor que $540^{\circ}$, ou seja, $180^{\circ}$ $<\mathrm{A}+\mathrm{B}+\mathrm{C}<540^{\circ}$; a soma dos lados de um triângulo esférico (perímetro) é menor que $360^{\circ}$.

Para resolver um triângulo esférico, há a necessidade de conhecer pelo menos três elementos (ângulos e distâncias). De posse destes elementos, basta aplicá-los nas fórmulas fundamentais da trigonometria esférica, para obter os demais. Com base na Figura 1, temos:

i) Fórmula dos quatro elementos:

Relativa a lados: Envolve três lados e um ângulo (Equação 1).

$\cos \mathrm{a}=\cos \mathrm{b} \cdot \cos \mathrm{c}+\operatorname{sen} \mathrm{b} \cdot \operatorname{sen} \mathrm{c} \cdot \cos \mathrm{A}(1)$ 
Relativa a ângulos: Envolve três ângulos e um lado (Equação 2).

$\cos A=\operatorname{sen} B \cdot \operatorname{sen} C \cdot \cos A-\cos B \cdot \cos C$

Analogia dos senos: Envolve dois lados e dois ângulos (Equação 3).

$(\operatorname{sem} a / \operatorname{sen} A)=(\operatorname{sen} b / \operatorname{sen} B)=$ $=(\operatorname{sen} \mathrm{c} / \operatorname{sen} \mathrm{C})$

Segundo Anton (2000) e Swokowski (1994), temos alguns conceitos sobre planos e comprimento de arco no espaço tridimensional:

Um plano no espaço 3-D pode ser determinado unicamente por um ponto específico no plano e o vetor perpendicular ao plano. Um vetor perpendicular ao plano é chamado de normal ao plano. Suponha uma equação do plano que passe pela origem em $\mathrm{P}_{0}$ $\left(\mathrm{x}_{0,} \mathrm{y}_{0}, \mathrm{z}_{0}\right)$ e é perpendicular ao vetor normal ao plano, $\mathbf{n}=(\mathrm{a}, \mathrm{b}, \mathrm{c})$. Pela Figura 2, sejam os vetores $\mathbf{r}_{0}=\left(\mathrm{x}_{0}, \mathrm{y}_{0}, \mathrm{z}_{0}\right)$ e $\mathbf{r}=(\mathrm{x}, \mathrm{y}, \mathrm{z})$ que o plano consiste precisamente dos pontos $\mathrm{P}=(\mathrm{x}, \mathrm{y}, \mathrm{z})$, para os quais o vetor $\mathbf{r}_{\mathbf{0}}-\mathbf{r}$ é ortogonal a $\mathbf{n}$, ou seja, $\mathbf{n} \cdot\left(\mathbf{r}_{\mathbf{n}}-\mathbf{r}\right)=0$.

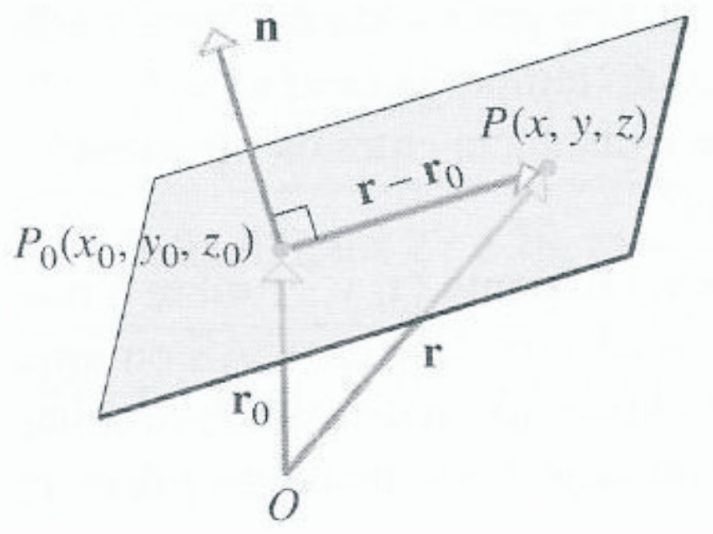

Figura 2. Plano ortogonal ao vetor $\mathbf{n}$.

Expressando esta equação vetorial em termos das componentes tem-se:

$(\mathrm{a}, \mathrm{b}, \mathrm{c}) \cdot\left(\mathrm{x}-\mathrm{x}_{0}, \mathrm{y}-\mathrm{y}_{0}, \mathrm{z}-\mathrm{z}_{0}\right)=0$, da qual obtém-se:

$\mathrm{a} \cdot\left(\mathrm{x}-\mathrm{x}_{0}\right)+\mathrm{b} \cdot\left(\mathrm{y}-\mathrm{y}_{0}\right)+\mathrm{c} \cdot\left(\mathrm{z}-\mathrm{z}_{0}\right)=0$, esta equação é chamada de forma ponto normal da equação de um plano. Efetuando-se a multiplicação, obtém-se a Equação 4:

$\mathrm{a} \cdot \mathrm{x}+\mathrm{b} \cdot \mathrm{y}+\mathrm{c} \cdot \mathrm{z}+\mathrm{d}=0$

Se $\mathbf{a}, \mathbf{b}, \mathbf{c}, \mathbf{d}$ forem constantes e $\mathbf{a}, \mathbf{b}, \mathbf{c}$ não forem todas nulas, então o gráfico da equação acima é um plano que tem a normal $\mathbf{n}=(\mathrm{a}, \mathrm{b}, \mathrm{c})$. Tem-se visto que um único plano é determinado por um ponto no plano e um vetor normal, não nulo ao plano. Em contrapartida, um plano não é determinado por um ponto no plano e um vetor paralelo, não-nulo, ao plano. Contudo um único plano é determinado por um ponto no plano e dois vetores não-paralelos que são paralelos ao plano, pois há um único plano que passa por P que é paralelo aos vetores $\mathbf{v}$ e w, como pode ser observado na Figura 3.

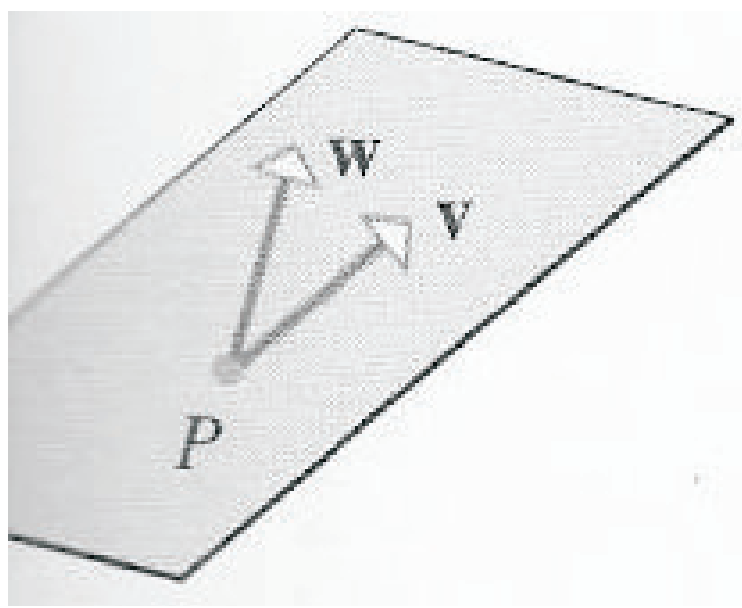

Figura 3. Plano que passa em $P$ que é paralelo aos vetores $\mathbf{v}$ e $\mathbf{w}$.

De acordo com Anton (2000), o comprimento de arco (L) de uma curva paramétrica $\mathrm{x}=\mathrm{x}(\mathrm{t}), \mathrm{y}=\mathrm{y}(\mathrm{t}), \mathrm{z}=\mathrm{z}(\mathrm{t}),(\mathrm{a} \leqslant \mathrm{t} \leqslant$ b) no espaço 3-D é dado pela Equação 5:

$$
L=\int_{a}^{b} \sqrt{\left(\frac{d x}{d t}\right)^{2}+\left(\frac{d y}{d t}\right)^{2}+\left(\frac{d z}{d t}\right)^{2}} d t
$$

Sistemas de coordenadas geográficas segundo o IBGE (1998):

Longitude geográfica $(\lambda)$ : é o ângulo medido ao longo do equador da Terra, tendo origem em um meridiano de referência (meridiano de 
Greenwich), e extremidade no meridiano do lugar. $\mathrm{Na}$ Conferência Internacional Meridiana, realizada em Washington em outubro de 1884, foi definida como variando de $0^{\circ} \mathrm{a}+180^{\circ}$ (Oeste de Greenwich) e de $0^{\circ}$ a $-180^{\circ}$ (Leste).

Latitude geográfica $(\varphi)$ : é o ângulo medido ao longo do meridiano do lugar, com origem no equador e extremidade no zênite do lugar. Varia entre $-90^{\circ}$ a $+90^{\circ}$. O sinal negativo indica latitudes do hemisfério sul e o sinal positivo, hemisfério norte.

Essas coordenadas podem ser observadas na Figura 4.

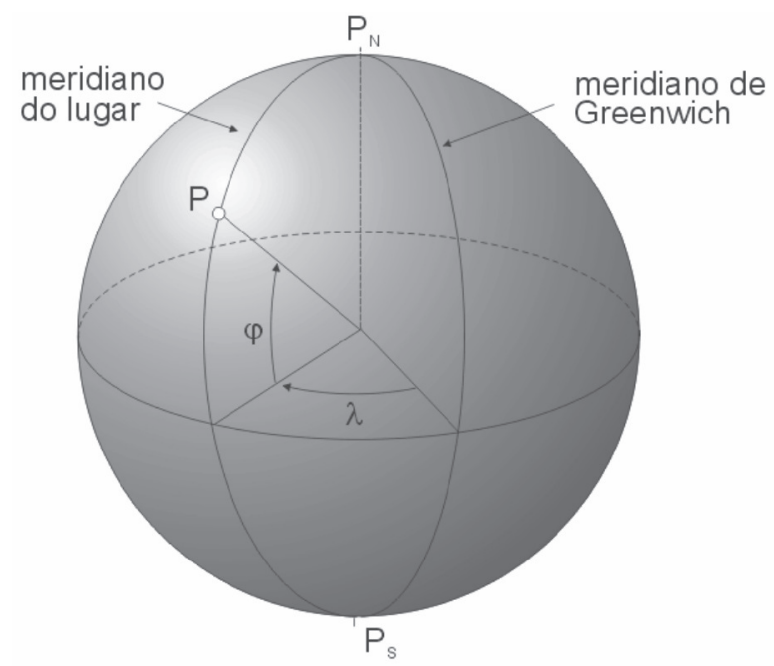

Figura 4. Coordenadas geográficas. Fonte. Wikipédia.

As coordenadas elipsoidais estão relacionadas com as coordenadas geográficas em latitude e longitude. Cada ponto sobre a superfície do elipsóide que tem como raio equatorial $=a$ e raio polar $=b$, tem-se coordenadas da forma $(\mathrm{d}, \varphi, \lambda)$, na qual $\varphi$ e $\lambda$ representam, respectivamente, a latitude e a longitude do ponto, e d representa a distância deste ponto ao centro do elipsóide (origem).

Convertendo essas coordenadas elipsoidais em coordenadas retangulares tridimensionais (x,y, z) (Equação 6), com origem no centro da Terra (Figura 5), o seu eixo $\underline{\mathbf{z}}$ positivo passando pelo Pólo Norte, o eixo $\underline{\mathbf{x}}$ positivo passando pelo meridiano de referência (Meridiano de Greenwich) e o eixo y positivo, satisfazendo-se a regra da mão direita (sistema dextrógiro) de acordo com a Figura 5.

$\mathrm{x}=a \cdot \cos (\varphi) \cdot \cos (\lambda)$
$\mathrm{y}=a \cdot \cos (\varphi) \cdot \operatorname{sen}(\lambda)$
$\mathrm{z}=b \cdot \operatorname{sen}(\varphi)$

Para saber a distância de um ponto sobre a superfície do elipsóide até a origem temos a Equação 7 :

$\mathrm{d}=\left(a^{2} \cdot \cos ^{2}(\varphi)+b^{2} \cdot \operatorname{sen}^{2}(\varphi)^{1 / 2}\right.$

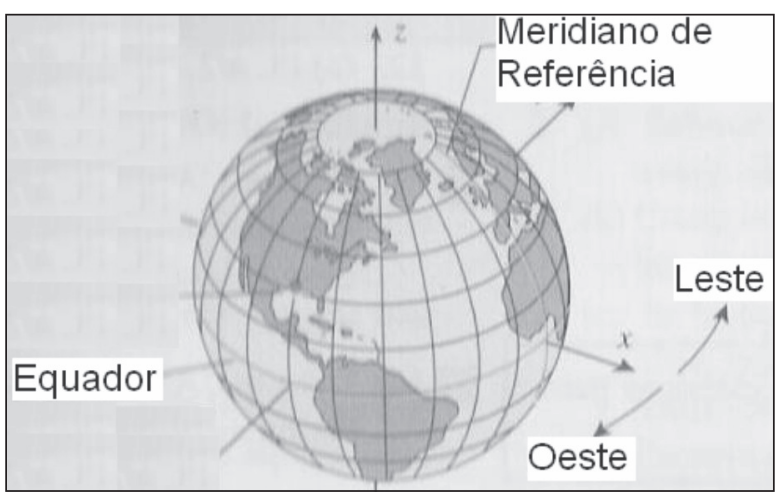

Figura 5. Coordenadas elipsoidais. Fonte: Adaptada de Anton (2000).

\section{MATERIAL E MÉTODOS}

Para os cálculos sobre o elipsóide foi utilizado o modelo adotado pelo Sistema Geodésico de Referência de 1980 - GRS80 (Geodetic Reference System 1980), que possui os seguintes parâmetros fundamentais:

$a=$ semi-eixo maior (raio equatorial) $=$ 6.378.137,0000 m;

$b=$ semi-eixo menor (raio polar) $=$ $6.356 .752,31414 \mathrm{~m}$;

$\alpha=$ achatamento $=1 / 298,257222101$;

$\omega=$ velocidade angular da Terra = $7.292 .115,0 \times 10^{-11} \mathrm{rad} / \mathrm{s}$;

$\mathrm{GM}=$ constante gravitacional da Terra $=$ $3.986 .004,418 \times 10^{8} \mathrm{~m}^{3} / \mathrm{s}^{2}$.

Para os cálculos sobre a esfera utilizouse um Raio Médio (RM), obtido pela Equação 8, segundo Espartel (1977):

$$
\mathrm{RM}=(2 \cdot a+b) / 3=6.371 .008,771 \mathrm{~m}(8)
$$


Para fazer este estudo foram escolhidas várias cidades distribuídas no globo terrestre, aplicando a elas várias rotas com distâncias aproximadas de 2.000 a $18.000 \mathrm{Km}$, e em várias direções, para se ter uma melhor e maior abrangência experimental.

Suas coordenadas geográficas aproximadas foram obtidas através do software MapSource (Tabela 1), com referência no sistema WGS-84.

Tabela 1. Coordenadas geográficas aproximadas das cidades.

\begin{tabular}{|c|c|c|}
\hline Cidades (País) & Latitude $(\varphi)$ & Longitude $(\lambda)$ \\
\hline Atenas (GRE) & $37^{\circ} 58^{\prime} 08^{\prime \prime} \mathrm{N}$ & $23^{\circ} 42^{\prime} 31^{\prime \prime} \mathrm{E}$ \\
\hline Bagdá (IRQ) & $33^{\circ} 20^{\prime} 36^{\prime \prime} \mathrm{N}$ & $44^{\circ} 24^{\prime} 24^{\prime \prime} \mathrm{E}$ \\
\hline Bankok (TAI) & $13^{\circ} 45^{\prime} 18^{\prime \prime} \mathrm{N}$ & $100^{\circ} 32^{\prime} 49^{\prime \prime} \mathrm{E}$ \\
\hline Berlin (ALE) & $52^{\circ} 28^{\prime} 54^{\prime \prime} \mathrm{N}$ & $13^{\circ} 17^{\prime} 36^{\prime \prime} \mathrm{E}$ \\
\hline Bogotá (COL) & $04^{\circ} 36^{\prime} 12^{\prime \prime} \mathrm{N}$ & $74^{\circ} 04^{\prime} 51^{\prime \prime} \mathrm{W}$ \\
\hline Buenos Aires (ARG) & $34^{\circ} 35^{\prime} 45^{\prime \prime} \mathrm{S}$ & $58^{\circ} 21^{\prime} 50^{\prime \prime} \mathrm{W}$ \\
\hline Cairo (EGI) & $30^{\circ} 03^{\prime} 21^{\prime \prime} \mathrm{N}$ & $31^{\circ} 14^{\prime} 13^{\prime \prime} \mathrm{E}$ \\
\hline Cidade do México (MEX) & $19^{\circ} 27^{\prime} 24^{\prime \prime} \mathrm{N}$ & $99^{\circ} 08^{\prime} 26^{\prime \prime} \mathrm{W}$ \\
\hline Havana (CUB) & $23^{\circ} 06^{\prime} 55^{\prime \prime} \mathrm{N}$ & $82^{\circ} 23^{\prime} 11^{\prime \prime} \mathrm{W}$ \\
\hline Honolulu (EUA) & $21^{\circ} 18^{\prime} 39^{\prime \prime} \mathrm{N}$ & $157^{\circ} 21^{\prime} 45^{\prime \prime} \mathrm{W}$ \\
\hline Jacarta (INS) & $06^{\circ} 09^{\prime} 48^{\prime \prime} \mathrm{S}$ & $106^{\circ} 49^{\prime} 12^{\prime \prime} \mathrm{E}$ \\
\hline Johanesburgo (AFS) & $26^{\circ} 12^{\prime} 09^{\prime \prime} \mathrm{S}$ & $28^{\circ} 03^{\prime} 33^{\prime \prime} \mathrm{E}$ \\
\hline Lagos (NIG) & $06^{\circ} 26^{\prime} 19^{\prime \prime} \mathrm{N}$ & $03^{\circ} 25^{\prime} 00^{\prime \prime} \mathrm{E}$ \\
\hline Londres (ING) & $51^{\circ} 30^{\prime \prime} 54^{\prime \prime} \mathrm{N}$ & $00^{\circ} 03^{\prime} 57^{\prime \prime} \mathrm{E}$ \\
\hline Los Angeles (EUA) & $34^{\circ} 06^{\prime} 45^{\prime \prime} \mathrm{N}$ & $118^{\circ} 24^{\prime} 38^{\prime \prime} \mathrm{W}$ \\
\hline Moscou (RUS) & $55^{\circ} 44^{\prime} 41^{\prime \prime} \mathrm{N}$ & $37^{\circ} 35^{\prime} 03^{\prime \prime} \mathrm{E}$ \\
\hline Nairobi (QUE) & $01^{\circ} 17^{\prime} 07^{\prime \prime} \mathrm{S}$ & $36^{\circ} 48^{\prime} 55^{\prime \prime} \mathrm{E}$ \\
\hline Nova Delhi (IND) & $28^{\circ} 34^{\prime} 32^{\prime \prime} \mathrm{N}$ & $77^{\circ} 12^{\prime} 03^{\prime \prime} \mathrm{E}$ \\
\hline Nova Venécia-ES (BRA) & $18^{\circ} 43^{\prime} 15^{\prime \prime} \mathrm{S}$ & $40^{\circ} 23^{\prime} 48^{\prime \prime} \mathrm{W}$ \\
\hline Nova York (EUA) & $40^{\circ} 43^{\prime} 36^{\prime \prime} \mathrm{N}$ & $73^{\circ} 59^{\prime} 35^{\prime \prime} \mathrm{W}$ \\
\hline Paris (FRA) & $48^{\circ} 51^{\prime} 22^{\prime \prime} \mathrm{N}$ & $02^{\circ} 20^{\prime} 24^{\prime \prime} \mathrm{E}$ \\
\hline Pequim (CHN) & $39^{\circ} 54^{\prime} 48^{\prime \prime} \mathrm{N}$ & $116^{\circ} 22^{\prime} 41^{\prime \prime} \mathrm{E}$ \\
\hline Rio de Janeiro (BRA) & $22^{\circ} 54^{\prime} 23^{\prime \prime} \mathrm{S}$ & $43^{\circ} 10^{\prime} 35^{\prime \prime} \mathrm{W}$ \\
\hline Roma (ITA) & $41^{\circ} 54^{\prime} 06^{\prime \prime} \mathrm{N}$ & $12^{\circ} 29^{\prime} 29^{\prime \prime} \mathrm{E}$ \\
\hline Santiago $(\mathrm{CHI})$ & $33^{\circ} 27^{\prime} 12^{\prime \prime} \mathrm{S}$ & $70^{\circ} 39^{\prime} 51^{\prime \prime} \mathrm{W}$ \\
\hline São Francisco (EUA) & $37^{\circ} 45^{\prime} 36^{\prime \prime} \mathrm{N}$ & $122^{\circ} 26^{\prime} 34^{\prime \prime} \mathrm{W}$ \\
\hline Seul (COR) & $37^{\circ} 33^{\prime} 44^{\prime \prime} \mathrm{N}$ & $126^{\circ} 58^{\prime} 48^{\prime \prime} \mathrm{E}$ \\
\hline Sydney (AUS) & $33^{\circ} 53^{\prime} 34^{\prime \prime} \mathrm{S}$ & $151^{\circ} 13^{\prime} 37^{\prime \prime} \mathrm{E}$ \\
\hline Tóquio (JAP) & $35^{\circ} 40^{\prime} 21^{\prime \prime} \mathrm{N}$ & $139^{\circ} 46^{\prime} 05^{\prime \prime} \mathrm{E}$ \\
\hline Viçosa-MG (BRA) & $20^{\circ} 45^{\prime} 40^{\prime \prime} \mathrm{S}$ & $42^{\circ} 52^{\prime} 10^{\prime \prime} \mathrm{W}$ \\
\hline Wellington (NZE) & $41^{\circ} 15^{\prime} 52^{\prime \prime} \mathrm{S}$ & $174^{\circ} 46^{\prime} 53^{\prime \prime} \mathrm{E}$ \\
\hline
\end{tabular}




\section{Distância no modelo esférico}

O método aplicado para o cálculo da distância esférica das rotas foi a fórmula dos quatro elementos, relativo a lados da trigonometria esférica (Figura 6), na Equação 9:

$\cos \alpha=\cos \beta \cdot \cos \gamma+\operatorname{sen} \beta \cdot \operatorname{sen} \gamma \cdot \cos A(9)$

sendo, $\beta=90^{\circ}-\varphi_{2} ; \gamma=90^{\circ}-\varphi_{1} ; A=\lambda_{2}-\lambda_{1}$ $\varphi_{1}=$ latitude do ponto 1

$\varphi_{2}=$ latitude do ponto 2

$\lambda_{1}=$ longitude do ponto 1

$\lambda_{2}=$ longitude do ponto 2

Considerando o raio médio da esfera de 6.371.008,771 m, tem-se:

$360^{\circ}-----2 \cdot \pi \cdot \mathrm{RM}=40.031,6124 \mathrm{~km}$.

$\alpha^{\circ}$--------- $d_{\text {esf }}=$ distância entre os dois pontos sobre a esfera (Equação 10).

$\mathrm{d}_{\mathrm{esf}}=\pi \cdot \mathrm{RM} \cdot \alpha^{\circ} / 180^{\circ}$

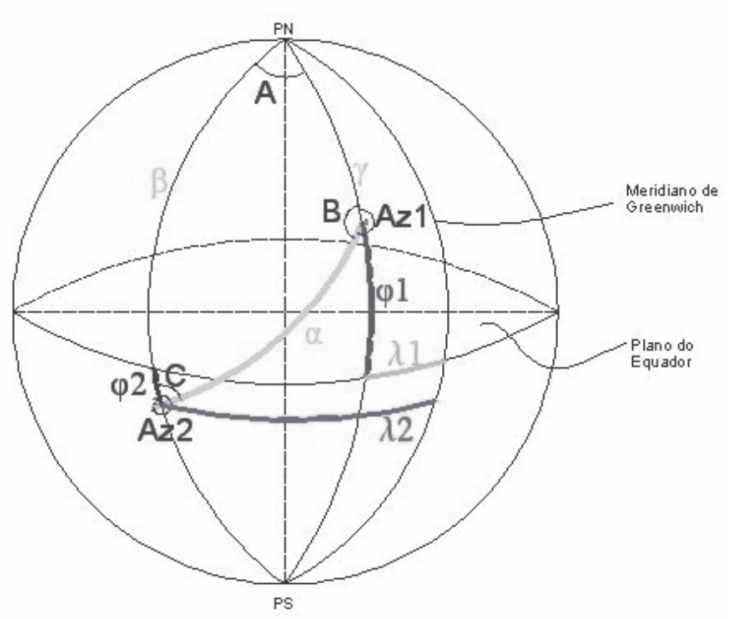

Figura 6. Modelo esférico.

\section{Distância no modelo elipsoidal}

Considerando um plano $\alpha$ que passe por dois pontos da superfície do elipsóide e pela sua origem (Figura 7). Tendo a equação do plano $\mathrm{a} \cdot \mathrm{x}+\mathrm{b} \cdot \mathrm{y}+\mathrm{c} \cdot \mathrm{z}+\mathrm{d}=0$ (Equação 4$)$, como o plano passa pela origem, $\mathrm{d}=0$.

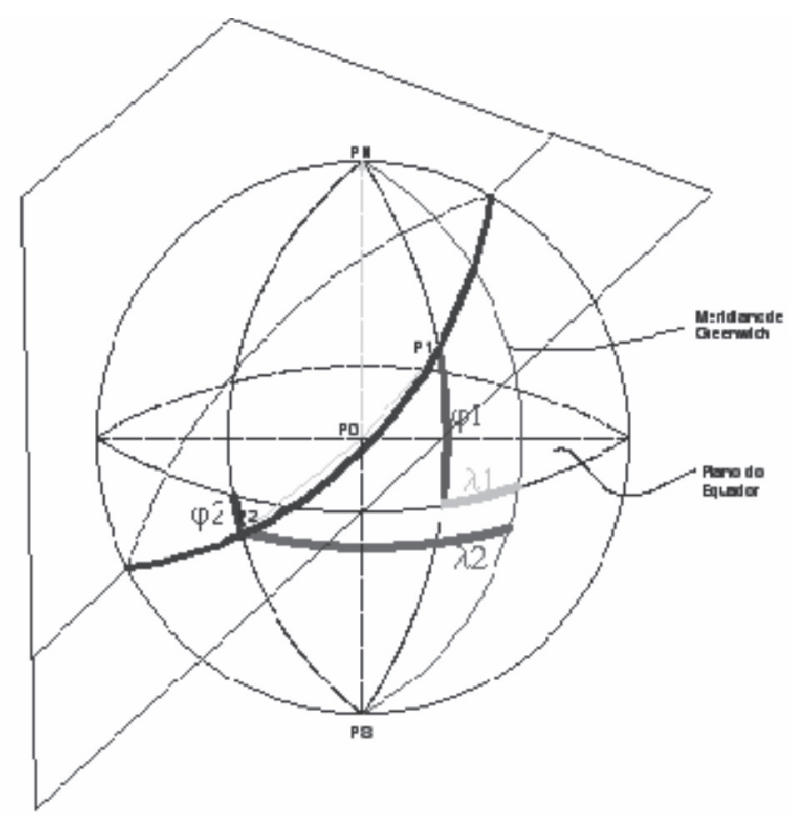

Figura 7. Interseção do plano com o elipsóide.

Assim, $a \cdot x+b \cdot y+c \cdot z=0$, isolandose uma das variáveis, $z=(-a / c) \cdot x+(-b / c) \cdot y$, substituindo-se $(-\mathrm{a} / \mathrm{c})$ e $(-\mathrm{b} / \mathrm{c})$ por $\mathbf{g}$ e $\mathbf{h}$ respectivamente tem-se a Equação 11:

$\mathrm{z}=\mathrm{g} \cdot \mathrm{x}+\mathrm{h} \cdot \mathrm{y}$

Com referência nas coordenadas retangulares de cada ponto sobre o elipsóide tem-se (12):

$\mathrm{x}=a \cdot \cos \varphi \cdot \cos \lambda$

$\mathrm{y}=a \cdot \cos \varphi \cdot \operatorname{sen} \lambda$

$\mathrm{z}=b \cdot \operatorname{sen} \varphi$

sendo, $a$ o raio equatorial e $b$ o raio polar.

A interseção do plano com o elipsóide dá-se o arco que une esses pontos, que é a menor distância sobre o elipsóide (Figura 7). Então, iguala-se a equação do plano com as equações das coordenadas retangulares do elipsóide, ou seja, substituindo (12) em (11) tem-se (13):

$b \cdot \operatorname{sen} \varphi=\mathrm{g} \cdot(a \cdot \cos \varphi \cdot \cos \lambda)+\mathrm{h} \cdot(a \cdot \cos \varphi \cdot \operatorname{sen} \lambda)$ $\operatorname{sen} \varphi=a \cdot \cos \varphi \cdot(\mathrm{g} \cdot \cos \lambda+\mathrm{h} \cdot \operatorname{sen} \lambda)$

$\operatorname{tg} \varphi=(a / b) \cdot(\mathrm{g} \cdot \cos \lambda+\mathrm{h} \cdot \operatorname{sen} \lambda)=\mathrm{M}(\lambda)$

(13) 
Para cada rota entre dois pontos temse um valor de g e h:

$\mathrm{P} 1: \mathrm{g} \cdot \cos \lambda_{1}+\mathrm{h} \cdot \operatorname{sen} \lambda_{1}=(b / a) \cdot \operatorname{tg} \varphi_{1}=\mathrm{K}\left(\varphi_{1}\right)$

$\mathrm{P} 2: \mathrm{g} \cdot \cos \lambda_{2}+\mathrm{h} \cdot \operatorname{sen} \lambda_{2}=(b / a) \cdot \operatorname{tg} \varphi_{2}=\mathrm{K}\left(\varphi_{2}\right)$

Multiplicando-se P1 por $\left(\cos \left(\lambda_{2}\right)\right)$ e P2 por $\left(\cos \left(\lambda_{1}\right)\right)$ e resolvendo o sistema, tem-se as Equações 14 e 15:

$$
\begin{array}{r}
\mathrm{h} \cdot\left(\operatorname{sen} \lambda_{1} \cdot \cos \lambda_{2}-\operatorname{sen} \lambda_{2} \cdot \cos \lambda_{1}\right)= \\
\downarrow \\
=(b / a) \cdot\left(\operatorname{tg} \varphi_{1} \cdot \cos \lambda_{2}-\operatorname{tg} \varphi_{2} \cdot \cos \lambda_{2}\right) \\
h=\frac{K\left(\varphi_{1}\right)^{*} \cos \lambda_{2}-K\left(\varphi_{2}\right)^{*} \cos \lambda_{1}}{\operatorname{sen} \lambda_{1} * \cos \lambda_{2}-\operatorname{sen} \lambda_{2} * \cos \lambda_{1}} \\
h=\frac{K\left(\varphi_{1}\right)^{*} \cos \lambda_{2}-K\left(\varphi_{2}\right)^{*} \cos \lambda_{1}}{\operatorname{sen}\left(\lambda_{1}-\lambda_{2}\right)} \\
\mathrm{g} * \cos \lambda_{1}=\mathrm{K}\left(\varphi_{1}\right)-\mathrm{h} * \operatorname{sen} \lambda_{1}, \\
g=\frac{K\left(\varphi_{1}\right)-K\left(\varphi_{1}\right)^{*} \cos \lambda_{2}-K\left(\varphi_{2}\right)^{*} \cos \lambda_{1}}{\cos \lambda_{1} * \operatorname{sen}\left(\lambda_{1}-\lambda_{2}\right)} \\
g=\frac{K\left(\varphi_{1}\right)^{*}\left[1-* \cos \lambda_{2}\right]-K\left(\varphi_{2}\right)^{*} \cos \lambda_{1}}{\cos \lambda_{1} * \operatorname{sen}\left(\lambda_{1}-\lambda_{2}\right)}
\end{array}
$$

$\operatorname{tg} \varphi=M(\lambda)$ da Equação 13, logo, pelo triângulo retângulo tem-se as Equações 16 e 17:

$$
\begin{aligned}
& \operatorname{sen} \varphi=\frac{M}{\sqrt{1+M^{2}}} \\
& \cos \varphi=\frac{1}{\sqrt{1+M^{2}}}
\end{aligned}
$$

Substituindo-se $\operatorname{tg} \varphi$, $\operatorname{sen} \varphi$, e $\operatorname{tg} \varphi$ nas equações das coordenadas (Equação12), para ficar somente em função de $\lambda$ tem-se as Equações 18, 19 e 20:

$$
\begin{aligned}
& x=\frac{\mathrm{a}^{*} \cos \lambda}{\sqrt{1+(\mathrm{a} / \mathrm{b})^{2} *(\mathrm{~g} * \cos \lambda+h * \operatorname{sen} \lambda)^{2}}} \\
& y=\frac{\mathrm{a}^{*} \operatorname{sen} \lambda}{\sqrt{1+(\mathrm{a} / \mathrm{b})^{2} *(\mathrm{~g} * \cos \lambda+h * \operatorname{sen} \lambda)^{2}}} \\
& z=\frac{\mathrm{b}^{*}(\mathrm{a} / \mathrm{b})^{*}(\mathrm{~g} * \cos \lambda+h * \operatorname{sen} \lambda)(20)}{\sqrt{1+(\mathrm{a} / \mathrm{b})^{2} *(\mathrm{~g} * \cos \lambda+h * \operatorname{sen} \lambda)^{2}}}
\end{aligned}
$$

Para calcular a distância entre esses dois pontos que situa no arco (Figura 7), aplicase na fórmula do comprimento de arco no espaço (Equação 21):

$$
d=\int_{\lambda_{\mathrm{L}}}^{\lambda_{2}} \sqrt{\left(\frac{d x}{d \lambda}\right)^{2}+\left(\frac{d y}{d \lambda}\right)^{2}+\left(\frac{d z}{d \lambda}\right)^{2}} d \lambda
$$

Para calcular as derivadas de $\mathrm{x}, \mathrm{y}$ e $\mathrm{z}$ em relação a $\lambda$ das Equações 18, 19 e 20 e a integral da Equação 21 foi utilizado o MAPLE 12, um software matemático. O intervalo de integração varia de $\lambda_{2}$ a $\lambda_{1}$, sendo que $\lambda_{2}>\lambda_{1}$, para o resultado não ser negativo.

Caso a longitude for constante, ou seja, os dois pontos estarem no mesmo meridiano. Neste caso, integra-se em função de $\varphi$ (Equação 22):

$$
d=\int_{\varphi_{1}}^{\varphi_{2}} \sqrt{\left(\frac{d x}{d \varphi}\right)^{2}+\left(\frac{d y}{d \varphi}\right)^{2}+\left(\frac{d z}{d \varphi}\right)^{2}} d \varphi
$$

\section{RESULTADOS E DISCUSSÃO}

As distâncias das rotas nos dois modelos podem ser observadas na Tabela 2, assim como os erros absolutos e erros relativos. 
Tabela 2. Distâncias das seguintes rotas nos modelos elipsoidal e esférico, com o erro absoluto e erro relativo.

\begin{tabular}{|l|c|c|c|c|}
\hline \multicolumn{1}{|c|}{ Rotas (de $\rightarrow$ para) } & D elip. $(\mathrm{Km})$ & D esf. (Km) & E abs. (Km) & E rel. (\%) \\
\hline 01) Atenas (GRE) $\rightarrow$ Berlin (ALE) & $1.803,539$ & $1.803,930$ & 0,391 & 0,022 \\
\hline 02) Paris (FRA) $\rightarrow$ Sydney (AUS) & $16.964,175$ & $16.964,373$ & 0,198 & 0,001 \\
\hline 03) Rio de Janeiro (BRA) $\rightarrow$ Roma (ITA) & $9.191,492$ & $9.198,017$ & 6,525 & 0,071 \\
\hline 04) Bogotá (COL) $\rightarrow$ Moscou (RUS) & $10.909,041$ & $10.907,574$ & 1,467 & 0,013 \\
\hline 05) Londres (ING) $\rightarrow$ São Francisco (EUA) & $8.633,450$ & $8.627,487$ & 5,963 & 0,069 \\
\hline 06) Nova Venécia-ES (BRA) $\rightarrow$ Johanesburgo (AFS) & $7.013,992$ & $7.006,881$ & 7,111 & 0,101 \\
\hline 07) Johanesburgo (AFS) $\rightarrow$ Buenos Aires (ARG) & $8.095,300$ & $8.087,840$ & 7,460 & 0,092 \\
\hline 08) Johanesburgo (AFS) $\rightarrow$ Sydney (AUS) & $11.046,776$ & $11.039,333$ & 7,443 & 0,067 \\
\hline 09) Cidade do México (MEX) $\rightarrow$ Nova Delhi (IND) & $14.659,483$ & $14.659,722$ & 0,239 & 0,002 \\
\hline 10) Nova York (EUA) $\rightarrow$ Pequim (CHN) & $10.991,587$ & $10.987,072$ & 4,515 & 0,041 \\
\hline 11) Tóquio (JAP) $\rightarrow$ Honolulu (EUA) & $6.248,803$ & $6.243,331$ & 5,472 & 0,088 \\
\hline 12) Sydney (AUT) $\rightarrow$ Santiago (CHI) & $11.349,513$ & $11.343,475$ & 6,038 & 0,053 \\
\hline 13) Honolulu (EUA) $\rightarrow$ Cidade do México (MEX) & $6.094,656$ & $6.088,093$ & 6,563 & 0,108 \\
\hline 14) Santiago (CHI) $\rightarrow$ Bogotá (COL) & $4.238,893$ & $4.246,968$ & 8,075 & 0,190 \\
\hline 15) Viçosa-MG (BRA) $\rightarrow$ Nova York (EUA) & $7.546,242$ & $7.556,204$ & 9,962 & 0,132 \\
\hline 16) Lagos (NIG) $\rightarrow$ Jacarta (INS) & $11.570,923$ & $11.558,568$ & 12,355 & 0,107 \\
\hline 17) Wellington (NZE) $\rightarrow$ Havana (CUB) & $12.717,592$ & $12.716,234$ & 1,358 & 0,011 \\
\hline 18) Bagdá (IRQ) $\rightarrow$ Seul (COR) & $7.248,195$ & $7.241,319$ & 6,876 & 0,095 \\
\hline 19) Bankok (TAI) $\rightarrow$ Cairo (EGI) & $7.279,644$ & $7.273,282$ & 6,362 & 0,087 \\
\hline 20) Los Angeles (EUA) $\rightarrow$ Nairobi (QUE) & $15.550,805$ & $15.548,372$ & 2,433 & 0,016 \\
\hline 21) Viçosa-MG (BRA) $\rightarrow$ Tóquio (JAP) & $18.329,032$ & $18.337,234$ & 8,202 & 0,045 \\
\hline
\end{tabular}

De acordo com a Tabela 2 podemos fazer algumas análises:

Rota 6) Dist. elip. > Dist. esf.: rota com predominância horizontal (paralelismo aos paralelos);

Rota 13) Dist. elip. > Dist. esf.: rota com predominância horizontal;

Rota 14) Dist. elip. < Dist. esf.: rota com predominância vertical (paralelismo aos meridianos);

Rota 15) Dist. elip. < Dist. esf.: rota com predominância vertical;

Rota 16) Dist. elip. > Dist. esf.: rota com predominância horizontal;

Rota 21) Dist. elip. < Dist. esf.: rota com predominância vertical.

A maior distância está na rota 21 $(>18.300 \mathrm{Km})$, com um erro absoluto de $8,202 \mathrm{Km}$, já a rota 16 apresenta uma distância menor $(>11.500 \mathrm{Km})$, e erro absoluto maior $(12,355 \mathrm{Km})$. Isto acontece devido ao fato da rota 21 ter sua trajetória próxima ao pólo norte e a rota 16 nas proximidades do equador.

A menor distância está na rota 1 $(>1.800 \mathrm{Km})$, com um erro absoluto de $391 \mathrm{~m}$, já a rota 2 apresenta uma distância maior ( $>$ $16.900 \mathrm{Km}$ ), e erro absoluto menor, sendo esta, a rota com o menor erro relativo entre todas as rotas $(0,001 \%)$. Isto se deve ao fato de a rota 2 ter sua trajetória com um ângulo próximo a $45^{\circ}$ em relação ao equador, provocando o efeito de compensação entre os raios equatorial $(a)$ e $\operatorname{polar}(b)$.

O maior erro relativo acontece na rota 14 (Santiago-CHI $\rightarrow$ Bogotá-COL) com 0,190\%. Apesar de a distância ser menor $\quad(>$ $4.200 \mathrm{Km}$ ) em relação a rota 21 , sua trajetória tem uma predominância vertical, ou seja, com um paralelismo ao meridiano. Com isso a distância no modelo elipsoidal é aproximadamente $8.000 \mathrm{~km}$ maior que no modelo esférico. 


\section{CONCLUSÃO}

Pode-se perceber que teve muitas variações para as distâncias no elipsóide e na esfera, ou seja, distâncias grandes com erro absoluto pequeno e distâncias pequenas com erro absoluto grande. Conclui-se que, as rotas onde acontecem as maiores variações de distâncias são as rotas com paralelismo aos meridianos e paralelos. Nas rotas com predominância vertical a distância sobre a esfera é maior que sobre o elipsóide e as com predominância horizontal a distância sobre o elipsóide é maior que sobre a esfera, já que o eixo equatorial é maior que o eixo polar. A confirmação é que os erros são significativos, pois 1' no círculo máximo equivale aproximadamente a um desvio de $1,852 \mathrm{~km}$.

Conclui-se que para aplicar essa metodologia na navegação oceânica e aérea, é recomendável utilizar os cálculos de distâncias sobre o elipsóide de revolução, pois é uma superfície matemática que melhor adapta-se a forma da Terra.

\section{REFERÊNCIAS BIBLIOGRÁFICAS}

Anton, H.; Cálculo, um novo horizonte; Trad. Cyro de Carvalho Patarra e Maria Tamanaha; $6^{\text {a }}$ ed. - Porto Alegre-RS: Bookman, Volume 2.2000.680p.

Espartel, L.; Curso de Topografia; $5^{\mathrm{a}}$ ed.Porto Alegre-RS: Editora Globo. 1977.658p.

Ferraz, A. S.; Elementos de Trigonometria Esférica; $1^{a}$ ed., UFV - Viçosa-MG: Imprensa Universitária, 1980.23p.

IBGE, Instituto Brasileiro de Geografia e Estatística, Diretoria de Geociências - DGC; Noções Básicas de Cartografia; Rio de Janeiro-RJ. 1998. 128p.
Swokowski, E. W.; Cálculo com geometria analítica; Trad. Alfredo Alves de faria; $2^{\mathrm{a}}$ ed. São Paulo-SP: Makron Books, Volume 2. 1994. 744p. 